SSLA, 21, 225-241. Printed in the United States of America.

\title{
LEXICAL PROCESSING STRATEGY USE AND VOCABULARY LEARNING THROUGH READING
}

\author{
Carol A. Fraser
}

York University

\begin{abstract}
This article reports on a strategy training study that investigated the lexical processing strategies (LPSs; ignore, consult, infer) used by $\mathrm{L} 2$ learners when they encounter unfamiliar vocabulary while reading and the impact of these strategies on vocabulary learning. A timeseries with repeated-measures design was used. Introspective data were gathered from eight participants (Francophone university students, intermediate ESL proficiency) on eight texts over 5 months to elicit LPS use on self-identified unfamiliar words. Then, 1 week after each reading, participants completed a cued recall task to measure their learning of these words. An analysis of overall LPS use (changes in patterns and effectiveness of strategy use with LPS-focused instruction) and word retention rates demonstrates the potential for vocabulary learning through reading and indicates that some LPSs lead to higher retention rates than others. This research increases our understanding of the role of LPS use in vocabulary learning and suggests some reevaluation of current pedagogic practice.
\end{abstract}

Although it is generally acknowledged in theory and research that some incidental L2 vocabulary learning occurs in the course of reading for comprehension, there is less consensus as to whether reading is a rich event for

I gratefully acknowledge support for this research from Educational Testing Services in the form of copies of the TOEFL Institutional Test and Glendon College, York University in the form of a Minor Research Grant. I also thank Esther Geva and Birgit Harley, who provided constructive feedback on an early draft of this paper. An earlier version of this paper was presented at the 11th World Congress of Applied Linguistics (AILA), Jyväskylä, Finland, August 1996.

Address correspondence to Carol Fraser, Glendon College, York University, English Department, 2275 Bayview Avenue, Toronto, Ontario, Canada M4N 3M6; e-mail: cfraser@erda.Glendon.yorku.ca. 
vocabulary learning. A variety of studies have found evidence of vocabulary growth (e.g., Huckin \& Bloch, 1993; Hulstijn, 1992; Paribakht \& Wesche, 1993, 1997; Pitts, White, \& Krashen, 1989), but views differ as to whether the demonstrated retention rates represent low (Hulstijn, 1992) or high (Krashen, 1989) learning outcomes.

Little is understood regarding how this incidental learning occurs, particularly with respect to the cognitive and metacognitive processes learners engage in when encountering unfamiliar words while reading and how differences in these processes affect vocabulary learning. The underlying assumption has been that incidental vocabulary learning primarily occurs through the process of inferring word meaning. But some research suggests that L2 learners who are left on their own generally ignore unfamiliar words, infer only when there is a specific need, and consult sparingly and on a very selective basis (Bensoussan \& Laufer, 1984; Paribakht \& Wesche, 1997). Because attention to an unknown word seems to be a prerequisite for any learning to occur (Ellis, 1994; Gass, 1988; Schmidt, 1994), high rates of ignoring would thus severely limit the learning potential.

Furthermore, there is a lack of consensus regarding the benefit for L2 learners of the use of lexical inference while reading. On the one hand, some researchers have argued that inferring word meanings is potentially a productive strategy for vocabulary learning. First, it is a communicative event in which learners engage in considerable hypothesis formation and testing about word meaning (Ellis, 1994; Haastrup, 1989, 1991) and, second, the rich psychological and linguistic context that text provides can act as a cognitive hook for the memory of new words (Schouten-van Parreren, 1989). However, researchers (Bensoussan \& Laufer, 1984; Haastrup, 1989, 1991; Haynes, 1984; Hulstijn, 1992) have also found that inferring is not always an easy or efficient strategy for L2 learners to use either because of text complexity (i.e., the text may not provide sufficient clues to infer) or because of reader limitations (i.e., L2 learners often do not make use of available cues or misuse them). Evidently, lexical inference is neither an efficient nor an effective vocabulary-learning strategy when L2 readers fail in their attempts to infer meaning for new words or when the meaning determined is inaccurate.

This article reports on a study of the lexical processing strategies that L2 learners use while reading for comprehension. Lexical processing strategies (LPSs) refer to the three strategic options an L2 reader has when confronting an unfamiliar word: ignore and continue reading, consult a dictionary or another individual, or infer word meaning on the basis of linguistic and contextual cues. Within the theoretical framework of classroom-based research, the goal of this study was, first, to describe the LPSs that L2 learners use while reading and, second, to trace the impact of LPS-focused instruction on LPS use and other relevant variables including reading rate, reading comprehension, and vocabulary learning. This paper presents results that address the issue of vocabulary learning and the role of lexical processing strategies. Spe- 
cifically, (a) Are the LPSs adult L2 learners use potentially productive for vocabulary learning? (b) Does LPS-focused instruction affect word learning? and (c) Are different word retention rates associated with reading proficiency, LPS use, or previous familiarity with the word?

\section{DESIGN OF THE STUDY}

Using a quasi-experimental, interrupted, time series with repeated-measures design, introspective and performance data were gathered individually from eight participants. All were Francophone university students enrolled in an intermediate level ESL course, "Academic Foundations," taught by the principal researcher.

There were two phases to the instructional treatment: metacognitive strategy training, followed by focused language instruction. Both phases were integrated into the regular content of the English for Academic Purposes (EAP) course involving 19 students. Each phase consisted of approximately 8 hours of directed instruction (4 in and 4 out of class) given over 1 month. Phase 1, metacognitive strategy training, focused on developing student awareness of the use and viability of the three LPS options. Overall, a direct instructional approach to strategy training was followed. This approach consisted of an explicit presentation of the LPSs (what the strategy is; why it is useful; and when, where, and how to use it), guided practice applying the strategy (teacher-student modeling and activities to apply outside class), and discussion of the results of the trial application (the effectiveness and efficacy of strategy use and problems encountered). In phase 2, instruction focused on building up the kinds of language knowledge thought to underlie the ability to use the LPSs-effective inferencing procedures, in particular. It included instruction on cognates, word structure (morphological word stems, prefixes, and suffixes), grammatical function (noun, verb, adjective, adverb, and conjunction), lexical cohesion (reiteration and collocation), and structural redundancy (appositive constructions, intersentential relations such as defining relative and adverbial clauses, and summary and rephrasing discourse markers). The presentation and practice of language items was contextualized and integrated into course reading tasks and focused on how learners could use this language information to determine the meaning of unfamiliar words.

The eight volunteer participants ${ }^{1}$ for the individual data collection sessions were selected to represent higher and lower levels of English reading proficiency on the basis of their results on the Vocabulary Levels Test (Nation, 1990) and a subsection of the Institutional TOEFL, Section 3, Vocabulary and Reading Comprehension (Educational Testing Service, 1992). These two tests were given to all students in the research class $(N=19)$. On the Vocabulary Levels Test, the class mean was 61.4 (maximum score 90; range 43-82; $S D$ 9.9). An analysis of class means for words at different frequency levels, according to test guidelines, indicated that vocabulary knowledge at the 2,000- and 
3,000-word frequency levels was acceptable but that this ESL group needed to develop more knowledge of words at the 5,000-and 10,000-word frequency levels. On the Vocabulary and Reading Comprehension Section of the TOEFL, the class mean was 46.4 (maximum score 67; range 39-53; SD 4.4). According to published performance references (Educational Testing Service, 1993), a mean of 46.4 for an undergraduate student is equivalent to a total scaled TOEFL score of about 490 . Class results on the two tests were highly correlated ( $r=$ .79). A scatter plot was used to differentiate the performance of participants on the two measures. Whereas the higher performance group scored above the mean on the Vocabulary Levels Test and above the score of 50 on the TOEFL (Section 3), those in the lower group scored below the class mean on the Vocabulary Levels Test and below 50 on the TOEFL (Section 3).

Participants met individually with the researcher nine times over 5 months for one training and eight data-collection sessions. These meetings represented four measurement periods: baseline, after metacognitive strategy training, after language-focused instruction, and a delayed measure given 1 month after the end of the instructional treatment to assess maintenance. In each data-collection session, participants first studied comprehension questions, read an article, answered comprehension questions, and skimmed the article to identify unknown words. They then engaged in an oral interview that focused on eliciting a retrospective think-aloud protocol of the LPSs they had used to deal with unfamiliar vocabulary while reading. A structured interview format (employing the same questions, in the same order, to all participants, for all texts) was used to increase the stability of the data in the repeatedmeasures design. All probes began with the general question "What did you do and think about when you first saw [the word] ' $x$ '?" followed by one to three more nondirective questions depending on the indicated LPS option. The texts were selected to be challenging; all came from the Science \& Technology section of The Economist and were 1,000-1,200 words long with a readability range of 9.4-12.3. ${ }^{2}$ Both a bilingual and an English dictionary were available for consultation.

Finally, 1 week after each reading, participants completed a cued recall task to monitor the effect of instruction on word learning. It consisted of 10 words selected from those each participant had indicated to be unknown and that had been focused upon in the retrospective think-aloud interview. Words were selected both to reflect a participant's frequency of use of the various LPS options and to ensure a balance of two levels of unknown words (i.e., either previously seen but not recalled or never seen before). Participants were asked to indicate their level of knowledge of the words using the 5-point Vocabulary Knowledge Scale (VKS, Paribakht \& Wesche, 1993; Wesche \& Paribakht, 1996). In this cued recall task, participants are asked to indicate their knowledge of words from (a) no familiarity to (b) recognition but no recall of meaning to (c) some recall (synonym or translation) to (d) use of the word in a sentence. 
Table 1. Frequency distribution of use of lexical processing strategies

\begin{tabular}{lcc}
\hline LPS option & Frequency & Percent \\
\hline Consult & 330 & 29 \\
Ignore & 269 & 24 \\
Infer & 491 & 44 \\
No attention & 37 & 3 \\
Total & 1127 & 100 \\
\hline
\end{tabular}

Note. "No attention" refers to the think-aloud protocols; participants occasionally indicated that they did not notice the unfamiliar word item while reading. The total is based on 878 unfamiliar word encounters.

\section{LEXICAL PROCESSING STRATEGY USE}

\section{Data Analysis}

Overall, 878 unknown words were coded and analyzed from the think-aloud protocol data (a maximum of 15 words per participant per text). Initially, frequency distributions based on total word encounters were examined to determine overall patterns of LPS use and the knowledge resources associated with those patterns. Subsequently, after confirmation that the distributions were normal using the K-S (Lilliefors) statistic, a multivariate repeated-measures analysis of variance was carried out on each of the variables for LPS use and success to investigate reading proficiency and instructional treatment effects. This analysis was based on each participant's rate of use of the different options and percentage of success at each time period. Additionally, orthogonal contrasts were used to break down the time effect into linear, quadratic, and cubic effects. $^{3}$

\section{Results}

LPS Use. Table 1 presents the overall use of the various lexical processing strategies. Participants used the three LPS options both alone and in combination with each other (e.g., infer and consult), which accounts for the 1,127 responses elicited on 878 word encounters. Furthermore, in 37 cases (3\%), participants reported that they had not noticed the unknown word while reading; these items were not analyzed further. Of the 841 remaining cases of actual strategy use, $71 \%$ represent single-LPS use and $29 \%$ represent multipleLPS use. As Table 1 indicates, overall, these participants inferred more frequently than they ignored or consulted.

Further examination of the frequency distributions associated with LPS use 


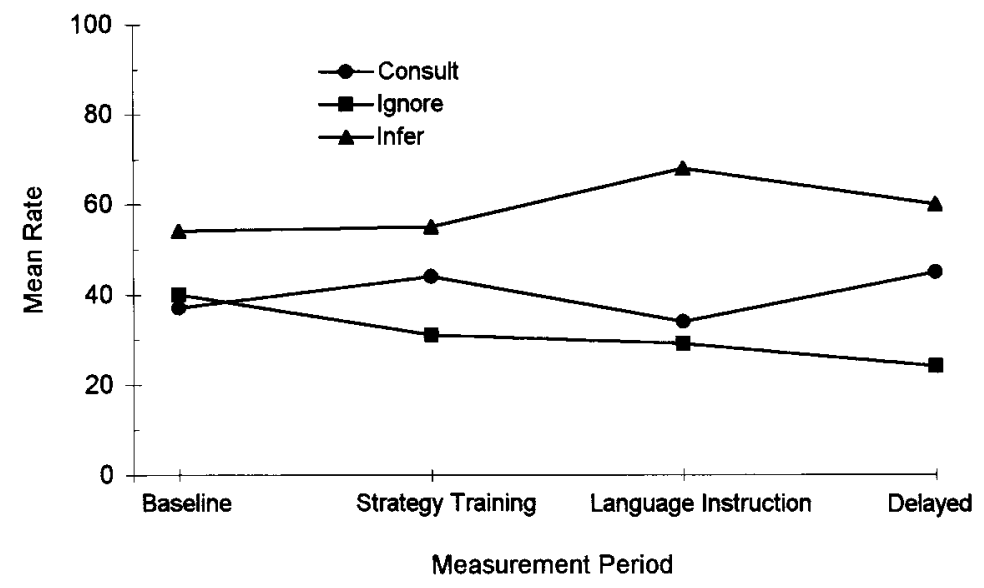

Figure 1. Mean rate of use of the three LPS options (consult, ignore, infer) by L2 readers $(N=8)$ over the four measurement periods. Rate of use is per 100 words. Rates do not add up to 100 because participants sometimes used LPS options in combination.

revealed that inferencing was the preferred as well as the primary strategy used by these participants. When the frequency of use presented in Table 1 is transformed into a rate of use based on the total number of words $(N=841)$ rather than on instance of LPS use $(N=1127)$, the outcome is that these learners inferred in $58 \%$ of the cases in which they encountered an unfamiliar word. Moreover, over half of these inferences occurred alone and, in contexts where inferencing was combined with other strategies, it was the first LPS option almost all of the time (96\%). In contrast, they consulted a dictionary $39 \%$ of the time-about half the time alone (55\%) and half the time following an inference (45\%) - and they ignored unfamiliar words $32 \%$ of the time, generally alone $(62 \%)$ but sometimes following an attempted inference (35\%).

Furthermore, this overall hierarchy of LPS use was maintained over time at all measures (Figure 1). That is, these L2 readers inferred more than they consulted and consulted more than they ignored. Although there were fluctuations and an overall slight increase in the rates of inferencing and consulting, the rate of ignoring decreased steadily. The repeated-measures ANOVA indicated that this decreasing linear trend for ignoring was significant, $F(3,4)=$ $12.97, p<.05$. There were no significant time effects for either inferencing or consulting.

Effectiveness of LPS Use. Each instance of LPS use in the protocol data was also rated in terms of how successful it was in determining a meaning for the word that was appropriate for text comprehension. A 3-point scale was used: no comprehension (i.e., no or an inappropriate meaning was determined), partial comprehension (i.e., the meaning determined worked generally for the text context although there was some distortion or loss of the text 


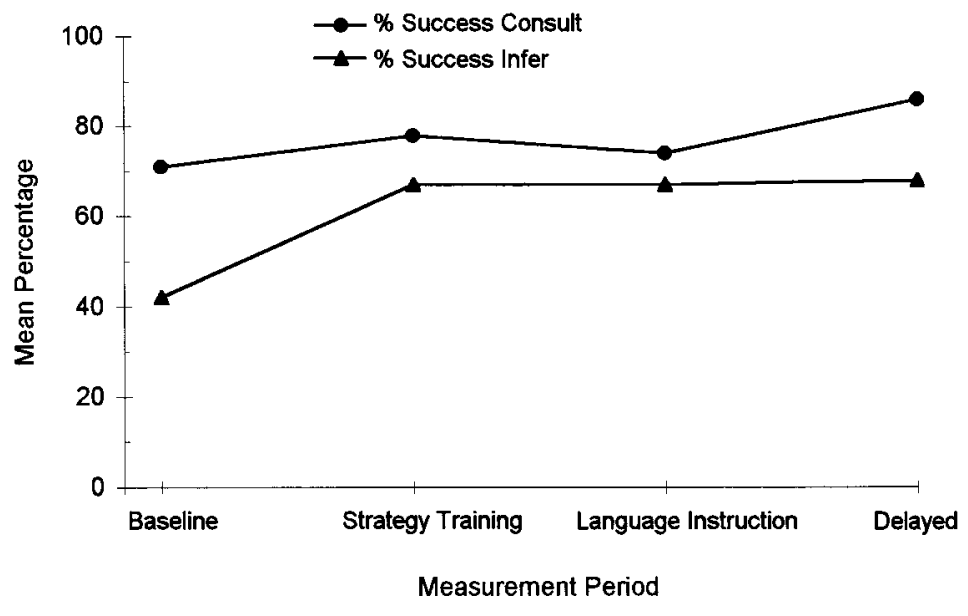

Figure 2. Mean success rates associated with the use of the LPS options, consult and infer, to determine appropriate word meaning for text comprehension over the four measurement periods.

representation, e.g., corpses as "bodies" instead of "dead bodies"), and comprehension (i.e., the meaning determined was appropriate for the text context with little or no meaning distortion). Each participant's rate of success when consulting and inferencing was computed by awarding a score of 0 for no comprehension, .5 for partial comprehension, and 1 for full comprehension.

An examination of the overall frequency distributions indicated that, when participants did consult or infer, they were generally successful; $78 \%$ of consults and $52 \%$ of inferences resulted in full comprehension and another $20 \%$ of inferences resulted in partial comprehension (i.e., the meaning determined worked adequately for text comprehension). Figure 2 further demonstrates that over all four time periods these participants were more successful in determining unfamiliar word meaning when consulting than when inferencing. More importantly, Figure 2 also illustrates a sharp increase in success when inferencing after the onset of metacognitive strategy training. The repeatedmeasures ANOVA indicated that this was a significant time effect, $F(3,4)=$ $7.80, p<.05$, which was both linear and quadratic.

Type and Effectiveness of Inference Processes Used. Additionally, all inferences were characterized according to the type of process involved. A distinction between word identification and sense creation processes previously made in word recognition research (Clark \& Gerrig, 1983; Kintsch \& Mross, 1985; Swinney 1979) was adopted. Inferencing through word identification is characterized as a fast, automatic, data-driven process in which the form of the unfamiliar word activates an L1 or L2 association in the learner's mental lexicon (e.g., stalking-talking; inherit-hériter and inhérant). The thematic context of the text plays a minimal role in word identification inferences. In the 
protocol data, such associations based on the phonological or orthographic form of the word seemed to "jump off the page" with the reader exerting little control over the association, as illustrated in these examples:

(1) T: What did you do and think about when you first saw "snapshots"?

S: Term of hockey.

$\mathrm{T}$ : What does it mean in hockey?

S: Just the snapshot, when someone shot in the goal. But here I didn't know the meaning so....

(2) T: What did you do and think about when you first saw "failure"?

S: I thought this is a kind of mistake or... It sounds like the same in French "faillir une émission" but I can't be sure.

In contrast, inferencing through sense creation is a context-centered, more deliberate and effortful process whereby meaning is created on the basis of language and situational cues from the text. As examples (3) and (4) illustrate, learners typically utilized both grammatical and situational cues from the immediate sentence context.

(3) T: What did you do and think about when you first saw "rotten"?

$\mathrm{S}$ : I knew that was a symptom of morning sickness, I know there was one was vomiting, and this one was probably, nausea or dizzy, so ... I didn't . . really find the real meaning because I knew it was a symptom.

(4) T: What did you do and think about when you first saw "soothe"?

S: It's the first time I see this word but I think it's the action to feed a baby.

T: How do you know?

S: Breast milk contains enough to feed a baby for a few hours so ...

An examination of the frequency distributions of LPS success by inference process type (Table 2) indicates that sense creation processes were used most frequently-that is, $65 \%$ of the time-and quite effectively, in that comprehension was achieved $78 \%$ of the time. Furthermore, even though L1 and L2 word identification processes were used equally (17\% and $18 \%$, respectively), these Francophone participants had more success using their L1 (77\% success) compared to their L2 (44\% success).

Figure 3 illustrates the use of inference process types over time and indicates that there was a steady increase in the proportion of inferences based on sense creation processes. In other words, these readers increasingly generated word meaning on the basis of linguistic and situational elements in the text rather than associating the unknown word with a phonologically or orthographically similar word in their L1 or L2 lexicons. Whereas the repeated-measures ANOVA indicated that this effect was approaching significance, $F(3,4)=$ $4.14, p<.10$, the univariate trend analysis indicated a significant linear trend, $F(1,6)=15.79, p<.01$. Over the course of the study, these L2 readers gradually increased the proportion of inferences based on sense creation processes but not to a point that reached statistical significance. 
Table 2. Frequency distribution of LPS success by inference process type

\begin{tabular}{lcccc}
\hline & \multicolumn{3}{c}{ Inference process type } & \\
\cline { 2 - 4 } \multicolumn{1}{c}{ LPS success } & $\begin{array}{c}\text { L1 word } \\
\text { identification }\end{array}$ & $\begin{array}{c}\text { L2 word } \\
\text { identification }\end{array}$ & Sense creation & All \\
\hline No comprehension & $23 \%$ & $56 \%$ & $22 \%$ & $28 \%$ \\
Partial & $17 \%$ & $9 \%$ & $23 \%$ & $20 \%$ \\
Complete & $60 \%$ & $35 \%$ & $55 \%$ & $52 \%$ \\
Total & $100 \%(n=83)^{\mathrm{a}}$ & $100 \%(n=87)$ & $100 \%(n=316)$ & $100 \%(n=486)$ \\
$\%$ use of process type & $17 \%{ }^{\mathrm{b}}$ & $18 \%$ & $65 \%$ & $100 \%$ \\
\hline
\end{tabular}

Note. The values represent percent of success in determining an appropriate meaning when inferring. No comprehension $=$ no or inappropriate meaning determined; Partial $=$ meaning determined worked generally for the text context with some loss or distortion; Complete = meaning determined was appropriate with little or no distortion. L1 word identification = inferring on the basis of L1 word association; L2 word identification = inferring on the basis of L2 word association; Sense creation = using linguistic and situational context to infer.

${ }^{a}$ Counts are in parentheses. ${ }^{b}$ These values represent percentage of use of each of the inference process types.

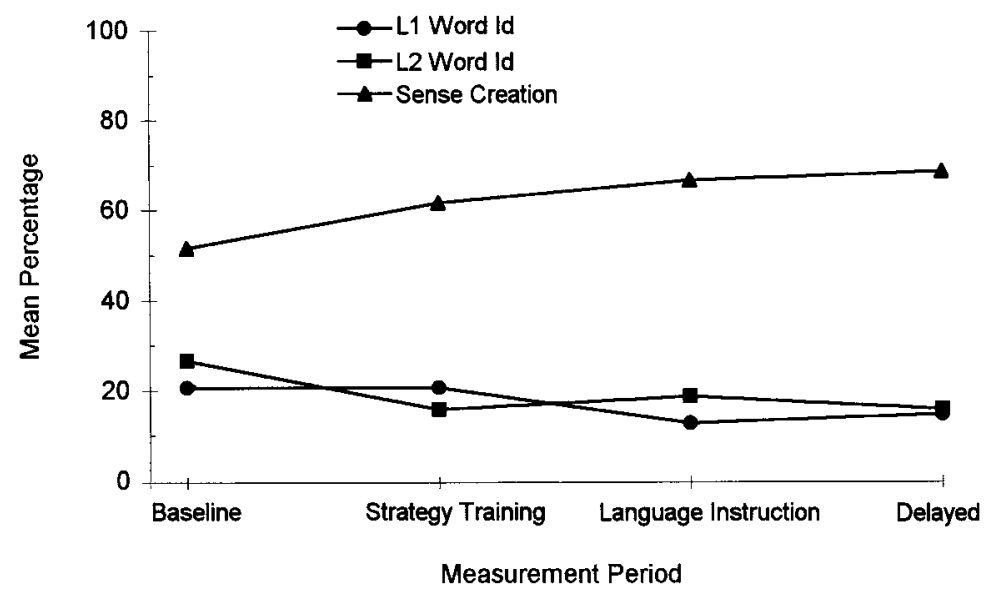

Figure 3. Mean rate of use of inference process types over the four measurement periods. L1 Word Id = use of L1 word association to infer; L2 Word Id = use of L2 word association to infer; Sense Creation = use of linguistic and situational context to infer.

Furthermore, if we examine the rate of success these learners had when using the various inference processes over time (Figure 4), we see that they became increasingly successful when inferencing with sense creation processes. The repeated-measures ANOVA indicated that this time effect was approaching significance, $F(3,4)=4.42, p<.10$. Also, this upward trend was both 


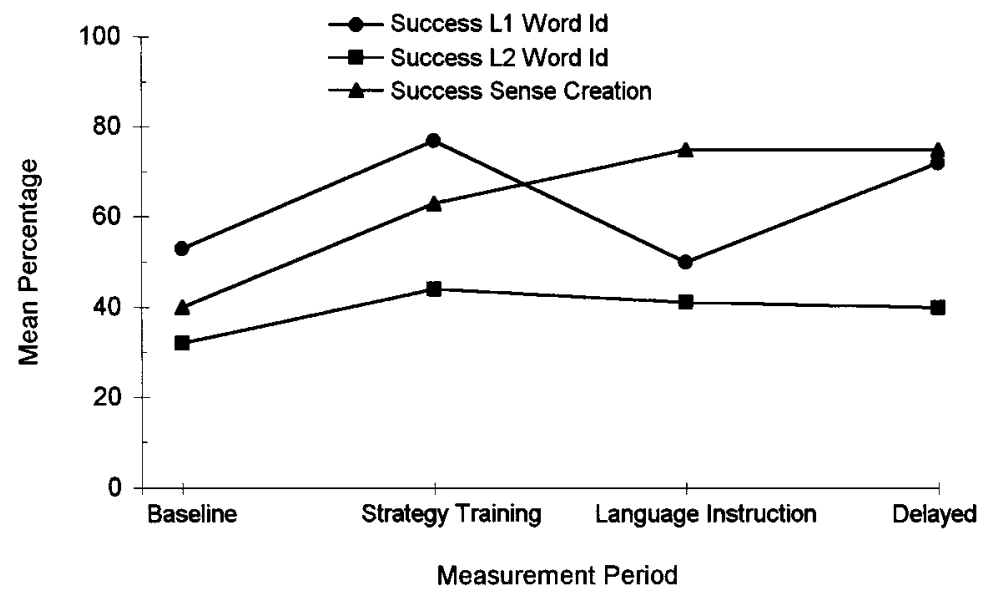

Figure 4. Mean success rates associated with the use of the various inference process types in determining appropriate word meaning for text comprehension over the four measurement periods.

linear, $F(1,6)=12.54, p<.05$, and quadratic, $F(1,6)=11.99, p<.05$, and was characterized by two distinct increases, one after the onset of metacognitive strategy training and one after the onset of language-focused instruction. Figure 4 also demonstrates that these Francophone learners had more success using their L1 than their L2 to determine word meaning. However, although the success rates using L2-based word-identification processes were quite stable, the success rates using L1-based word-identification processes fluctuated dramatically.

\section{LEXICAL PROCESSING STRATEGY USE AND VOCABULARY LEARNING}

\section{Data Analysis}

The VKS, which was the format used in the cued recall task to monitor the effect of LPS instruction on vocabulary learning, uses a 5-point scale to distinguish different levels of a learner's knowledge of a word: 1 (not familiar), 2 (familiar but meaning is not known), 3 (correct synonym or translation is given), 4 (word is used with semantic appropriateness in a sentence), and 5 (word is used with grammatical and semantic appropriateness in a sentence). Initially, all items were scored according to the VKS. However, because of the low frequency of use of the categories distinguishing intermediate levels of knowledge for analysis (i.e., categories 1, 4, and 5), the 5-point VKS score was collapsed to a 2-point score (meaning recalled vs. not recalled). Additionally, because the goal of this word learning measure was to track retention associated with LPS use, the rating of participants' learning of a word was based on 


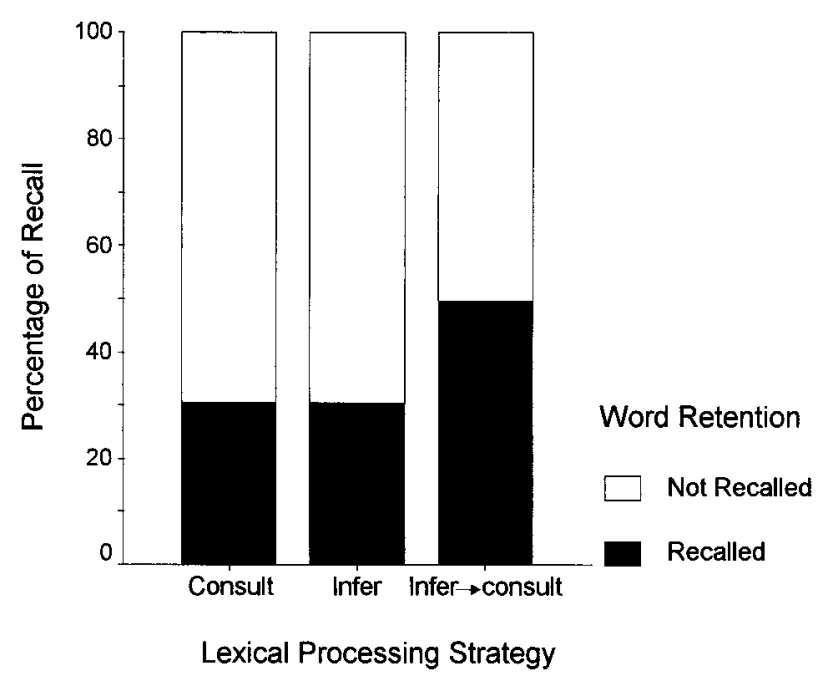

Figure 5. Percentage of words recalled according to LPS use by L2 readers $(N=8)$.

the meaning determined by participants in their initial LPS use and not a standard meaning of the word. For example, through LPS use, one participant determined that sights meant taille ("size") and, subsequently, in the cued recall task supplied the translation taille to the prompt sights; when scored, she was awarded a score of 3 (correct synonym or translation is given) even though the meaning determined was incorrect.

A total of 622 cued recall items were analyzed. ${ }^{4}$ The word-learning score for each time period represents the percentage of demonstrated retention of words over two texts (i.e., 20 words). A multivariate repeated-measures ANOVA was carried out to examine the effects of instruction and reading proficiency level on word learning scores. Additionally, frequency distributions were analyzed to examine patterns of word retention associated with LPS use, inference process type, and level of familiarity with the word.

\section{Results}

Word-learning scores for the four time periods had a range of $5-50 \%$ and an overall mean of $28 \%(S D=12)$; individual participant means ranged from $13 \%$ to $40 \%$. Results of the repeated-measures ANOVA indicated no significant main effects for group (i.e., L2 reading proficiency) or time (i.e., instruction), and no group-by-time interaction effect.

The analysis of the frequency distributions of word-learning scores associated with LPS option, inference process type, and level of word familiarity indicated the following patterns. First (see Figure 5), when participants consulted or inferred alone, they recalled the word meaning they had deter- 


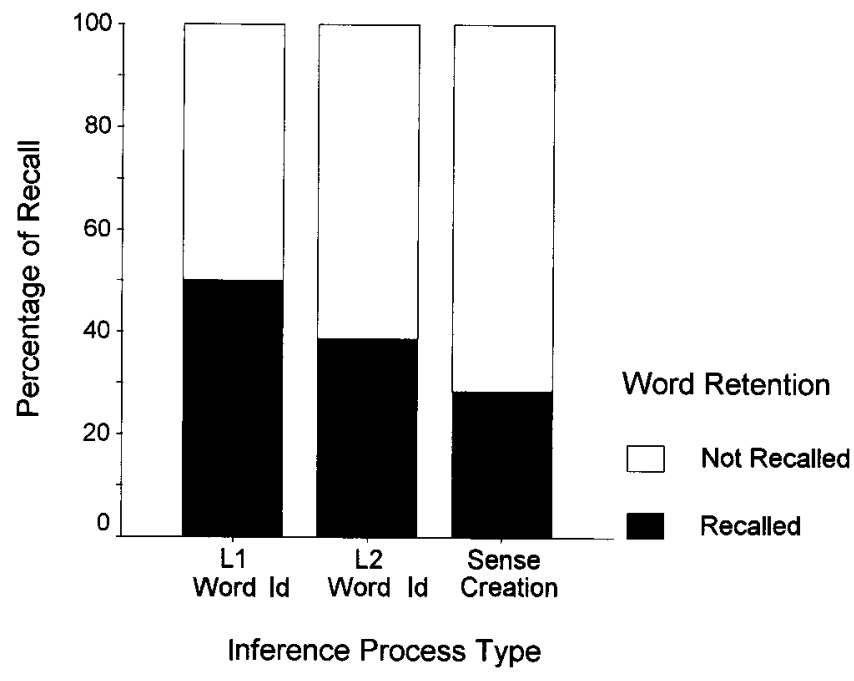

Figure 6. Percentage of words recalled according to type of inferencing process by L2 readers $(N=8)$.

mined about $30 \%$ of the time (30\% and $31 \%$, respectively). However, when they inferred and then consulted, their recall increased to $50 \%$.

Second (see Figure 6), when participants used L1-based word-identification processes to infer, they had a mean retention rate of $50 \%$, which decreased to $39 \%$ for L2-based word-identification inferences and $28 \%$ for sense creation inferences.

Third (Figure 7), word retention rates were higher for words that participants initially indicated they had previously seen but could not recall (42\%) compared to words they had not seen before (25\%).

\section{DISCUSSION AND CONCLUSION}

This article has reported on results from a study of the lexical processing strategies L2 readers use while reading. The results support the view that reading for comprehension in an L2 can be productive for incidental vocabulary learning. When dealing with unfamiliar vocabulary, these L2 learners used LPSs that were productive for word learning (i.e., consulting and inferencing) more frequently than unproductive ones (i.e., ignoring or not paying attention). They were also generally successful in determining a meaning that was adequate for text comprehension when both consulting and inferencing.

Moreover, when inferencing, they accessed sense creation processes that made use of cues from the text context more frequently than word-identification processes that relied on L1 or L2 form-based associations. Previous research in L2 inferencing (Bensoussan \& Laufer, 1984; Haynes, 1984; Huckin \& 


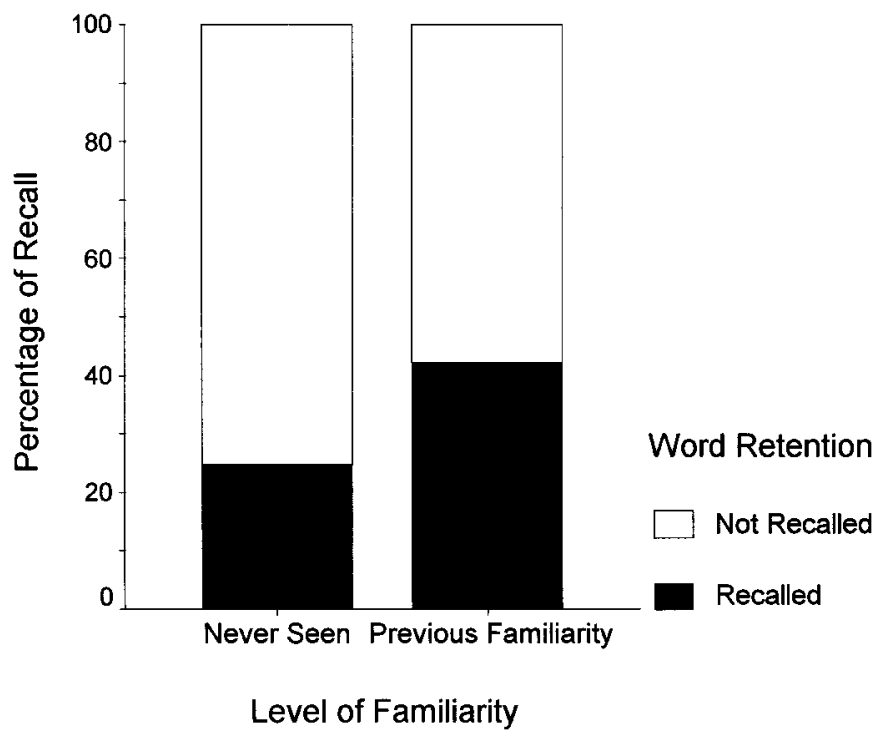

Figure 7. Percentage of words recalled according to level of previous familiarity by L2 readers $(N=8)$. The level of familiarity represents participants' perception of their knowledge: either never seen before or previously seen but not recalled.

Bloch, 1993) has indicated that inferences based on word form associations rather than the use of cues from the text context often lead to inappropriate word-meaning determination. The potential for L1 cognate associations to be especially misleading was highlighted in this study by the dramatically fluctuating success rates of the learners when inferencing using L1-based word-identification processes.

Although instruction focusing on enhancing lexical processing strategy use did not have a direct effect on vocabulary learning, there was evidence of an indirect effect. First, the rate of ignoring words decreased with instruction, and success when making inferences increased. This increase in the effectiveness of the inferencing strategy was related to steady increases in both the rate of use and percentage of success these L2 readers had when inferencing using sense creation processes. In other words, not only did these learners increase the number of inferences that were based on the linguistic and situational context of the text, but these inferences became increasingly appropriate. The LPS-focused instruction thus seemed to improve the underlying conditions needed for effective vocabulary learning while reading: More unfamiliar words were attended to, more extensive and elaborative processing was undertaken, and more appropriate meanings were determined.

With respect to vocabulary learning, substantial individual differences were indicated by the wide range of word retention scores across participants 
(5-50\%) and the high standard deviation (12). Differences in L2 reading proficiency level did not affect the retention of the meaning of new words encountered while reading. Sanaoui (1992) reported a similar finding in a study that investigated the vocabulary learning of adult L2 (French) learners at different language-proficiency levels.

Word-retention scores on the cued recall task indicate that these learners did acquire vocabulary while reading for meaning. ${ }^{5}$ Furthermore, the mean retention rate of $28 \%$ is considerable in the light of previous research. For example, in the context of a single encounter with a new word while reading, with no prompt to infer, both Krashen (1989) and Hulstijn (1992) reported retention rates of about $7 \%$. In the context of multiple encounters with target words with no prompt to infer, Paribakht and Wesche (1997) reported a retention rate of $11 \%$ for verbs and $24 \%$ for nouns, and, in a single reading in which the inferring process was prompted and guided, Hulstijn (1992) reported retention rates of $13 \%$ and $17 \%$.

The higher retention rates in this study could relate to several factors. First, the words tested for retention were self-selected by the learners in the sense that they initially identified these words as unfamiliar. These words thus represented a corpus of unknown words that were both noticed and potentially highly salient for these learners (e.g., partially known words, L1 cognates, and words encountered multiple times). Additionally, the retrospective think-aloud interview may in itself have constituted an added word-learning opportunity. Minimally, participants' attention was focused on the orthographic and phonological form of each word, but participants also retrieved, rehearsed, and elaborated earlier thought processes on the semantic and syntactic features of the words. This factor could have allowed for more explicit learning involving a higher level of awareness than might normally occur in reading and thus may have enhanced retention (Ellis, 1994; Pressley \& Afflerbach, 1995).

Finally, this study found evidence that some LPSs were associated with higher retention rates than others. Predictably, these learners had higher retention rates when an inference was based on an already existing L1 or L2 word association. There was already a cognitive hook to hang the new item on. This facilitating effect of existing memory structures was also seen in the higher retention rates for words with which learners had some previous familiarity compared to those reported as never before seen. This supports the view that vocabulary is learned incrementally over multiple encounters (Nagy, Herman, \& Anderson, 1985).

Furthermore, when these learners inferred and then consulted, they had a higher retention rate than if they inferred or consulted alone. Schouten-van Parreren (1989) reported a similar finding in her case study of adult L2 learners. Inferring unknown word meaning and then consulting a dictionary for verification may thus be a routine that involves a greater depth of processing, that is, more rehearsal and more elaboration, than either inferring or consulting alone and, accordingly, results in enhanced retention (Craik \& Lockhart, 
1972). Furthermore, as Kelly (1990) noted, this is also a routine that can, to a significant extent, offset the risk of inferring an incorrect meaning.

In conclusion, this research adds to our understanding of incidental L2 vocabulary growth through reading and also provides some insight into how the lexical processing strategies L2 learners use when encountering unfamiliar vocabulary affect vocabulary learning. It suggests that, although instruction that enhances lexical processing strategy use does not directly affect vocabulary learning, it can have an indirect effect by decreasing the number of unfamiliar words ignored and increasing the quality of inferences.

\section{Instructional Implications}

This research supports the efficacy of instruction that aims to improve L2 learners' ability to infer new word meanings and provides specific direction regarding the kind of instruction on inferencing that is appropriate. Overall, it supports the instructional focus outlined by Nation (1990) whereby students are provided with a strategic procedure that guides them to use elements in the immediate sentence context, first as the basis for their inference and, subsequently, to monitor its appropriateness.

However, the results of this study also bring into question Nation's (1990) recommendation to direct students to avoid using word form cues until after they have inferred on the basis of sentence context. Nation's rationale for sequencing the use of word form cues to the end as a monitoring procedure was that research had consistently shown that a common cause of error in lexical inferencing was use of the word form rather than the context to infer. Data from this study also support this observation. Additionally, however, results suggest that the use of word form cues are typically accessed in a fast, automatic manner as part of the process of inferencing through word-identification processes. Other researchers have also noted the primacy of word form cues in the inferencing process (Bensoussan \& Laufer, 1984; Haynes, 1984; Huckin \& Bloch, 1993). Accordingly, it seems unlikely that we can train students to hold off their potentially automatic use of word form cues. Rather, instruction needs to guide students to monitor and elaborate inferences based on wordidentification processes through the use of the immediate sentence and the wider text context.

Results from this study highlight the importance of consulting as an explicit vocabulary-learning strategy, particularly when it is used to verify an inference. Thus, this study suggests a reevaluation of the minimal role often accorded to dictionary use in the L2 reading class. Results accentuate the importance of consulting both for effective determination of word meaning and for vocabulary learning. This finding is consistent with research that has found that consulting a dictionary enhances the reading comprehension (Knight, 1994) and vocabulary learning (Knight, 1994; Luppescu \& Day, 1993) of L2 learners. In the present study, the use of consulting was an area of great individual difference in both the frequency and effectiveness of its use. Several 
participants rarely consulted and one participant had continuing difficulty accessing an appropriate meaning when consulting the dictionary. Therefore, there may be a need to focus in the classroom on the efficient and effective use of a dictionary while reading.

A final pedagogical implication is that L2 learners will likely benefit from instruction that focuses on developing strategies for acquiring vocabulary through reading. Results indicated that the learners in this study did acquire a considerable amount of vocabulary in the context of a single reading of a text for meaning. Moreover, some learners demonstrated substantially higher rates of retention than others, and some LPSs were associated with higher retention rates than others. Direct strategy training-the kind that makes students aware of the differential impact of various LPS routines on vocabulary learning and that introduces them to heuristics that facilitate the development of rich and elaborated memory traces for words-should enable L2 learners to more fully exploit the rich potential reading provides for vocabulary learning.

\section{NOTES}

1. The eight student participants received an honorarium.

2. Readability was assessed using the Readability Estimator (Berta-Max Educational Software, 1988). A readability average was determined on the basis of five readability formulas (The Dale-Chall Index, The Fog Index, The Flesch Index, The Flesch-Kincaid Index, and The Fry Index).

3. Because of the small number of participants, the power of analysis was low. Accordingly, although an alpha level of .05 was used to assess statistical significance, effects that were approaching statistical significance, that is, an alpha level of .10, were examined as well. This was done to offset the risk that important, though statistically nonsignificant, effects would be overlooked (see Borg, 1987; Pressley \& Afflerbach, 1995).

4. From the total of 640 cued recall items collected, 18 were not analyzed. Occasionally, participants did not indicate 10 unfamiliar words in a text. To ensure that all vocabulary learning measures consisted of 10 recall items, the researcher selected supplementary items (18 in total), which were subsequently deleted from the data base.

5 . As one reviewer noted, these remembered word meanings could be approximate and even incorrect. However, if vocabulary learning is viewed as a continuous and incremental process, then the vocabulary knowledge that any individual has at a given time is never fully complete and accurate (De Bot, Paribakht, \& Wesche, 1997; Nagy \& Herman, 1987). Within this framework, in the present study vocabulary learning occurred to the extent that participants remembered the meanings that they had determined for unfamiliar words encountered in the course of reading. Even though these meanings could be partial and inaccurate, participants' memory of them demonstrates learning. The quality or accuracy of this vocabulary learning was examined through the analysis of the success or effectiveness of participants' use of the various lexical processing strategies for determining appropriate word meaning for text comprehension.

\section{REFERENCES}

Bensoussan, M., \& Laufer, B. (1984). Lexical guessing in context in EFL reading comprehension. Journal of Reading Research, 7, 15-32.

Berta-Max Educational Software. (1988). Readability estimator. Anacortes, WA: Berta-Max, Inc.

Borg, W. R. (1987). Applying educational research. London: Longman.

Clark, H. H., \& Gerrig, R. J. (1983). Understanding old words with new meanings. Journal of Verbal Learning and Verbal Behavior, 29, 591-608.

Craik, F. I. M., \& Lockhart, R. R. (1972). Levels of processing: A framework for memory research. Journal of Verbal Learning and Verbal Behaviour, 11, 671-684.

De Bot, K., Paribakht, T. S., \& Wesche, M. B. (1997). Toward a lexical processing model for the study 
of second language vocabulary acquisition: Evidence from ESL reading. Studies in Second Language Acquisition, 19, 309-329.

Educational Testing Service. (1992). The Test of English as a Foreign Language. Princeton, NJ: Educational Testing Service.

Educational Testing Service. (1993). TOEFL: Institutional Testing Program. Princeton, NJ: Educational Testing Service.

Ellis, N. C. (1994). Consciousness in second language learning: Psychological perspectives on the role of conscious processes in vocabulary acquisition. AILA Review, 11, 37-56.

Gass, S. (1988). Integrating research areas: A framework for second language studies. Applied Linguistics, 9, 198-217.

Haastrup, K. (1989). The learner as word processor. AILA Review, 6, 34-46.

Haastrup, K. (1991). Lexical Inferencing Procedures or Talking About Words. Tübingen: Gunter Narr.

Haynes, M. (1984). Patterns and perils of guessing in second language reading. In J. Handscombe, R. A. Orem, \& B. P. Taylor (Eds.), On TESOL '83 (pp. 163-176). Washington, DC: TESOL Publication.

Huckin, T., \& Bloch, J. (1993). Strategies for inferring word meaning in context: A cognitive model. In T. Huckin, M. Haynes, \& J. Coady (Eds.), Second language reading and vocabulary learning (pp. 153-176). Norwood, NJ: Ablex.

Hulstijn, J. H. (1992). Retention of inferred and given word meanings: Experiments in incidental vocabulary learning. In P. J. L. Arnaud \& H. Béjoint (Eds.), Vocabulary and applied linguistics (pp. 113-125). London: Macmillan.

Kelly, P. (1990). Guessing: No substitute for systematic learning of lexis. System, 18, $199-207$.

Kintsch, W., \& Mross, E. F. (1985). Context effects in word identification. Journal of Memory and Language, 24, 336-349.

Knight, S. (1994). Dictionary use while reading: The effects on comprehension and vocabulary acquisition for students of different abilities. The Modern Language Journal, 78, 285-299.

Krashen, S. (1989). We acquire vocabulary and spelling by reading: Additional evidence for the input hypothesis. The Modern Language Journal, 73, 443-463.

Luppescu, S., \& Day, R. R. (1993). Reading dictionaries and vocabulary learning. Language Learning, 43, 263-287.

Nagy, W. E., \& Herman, P. A. (1987). Breadth and depth of vocabulary knowledge: Implications for acquisition and instruction. In M. G. McKeown \& M. E. Curtis (Eds.), The nature of vocabulary acquisition (pp. 19-35). Hillsdale, NJ: Erlbaum.

Nagy, W. E., Herman, P. A., \& Anderson, R. C. (1985). Learning words from context. Reading Research Quarterly, 20, 233-253.

Nation, I. S. P. (1990). Teaching and Learning Vocabulary. Rowley, MA: Newbury House.

Paribakht, T. S., \& Wesche, M. (1993). Reading comprehension and second language development in a comprehension-based ESL program. TESL Canada Journal, 11, 9-29.

Paribakht, T. S., \& Wesche, M. (1997). Vocabulary enhancement activities and reading for meaning in second language vocabulary acquisition. In J. Coady \& T. Huckin (Eds.), Second language vocabulary acquisition: A rationale for pedagogy (pp. 174-200). New York: Cambridge University Press.

Pitts, M., White, H., \& Krashen, S. (1989). Acquiring second language vocabulary through reading: A replication of the Clockwork Orange study using second language acquirers. Reading in a Foreign Language, 5, 271-275.

Pressley, M., \& Afflerbach, P. (1995). Verbal protocols of reading: The nature of constructively responsive reading. Hillsdale, NJ: Erlbaum.

Sanaoui, R. (1992). Vocabulary learning and teaching in the French as a second language classroom. Unpublished doctoral dissertation, University of Toronto (OISE).

Schmidt, R. (1994). Deconstructing consciousness in search of useful definitions for applied linguistics. AlLA Review, 11, 11-26.

Schouten-van Parreren, C. (1989). Vocabulary learning through reading: Which conditions should be met when presenting words in texts? AILA Review, 6, 75-85.

Swinney, D. A. (1979). Lexical access during sentence comprehension: (Re)consideration of context effects. Journal of Verbal Learning and Verbal Behaviour, 18, 645-659.

Wesche, M., \& Paribakht, T. S. (1996). Assessing second language vocabulary: Depth versus breadth. The Canadian Modern Language Review, 53, 13-40. 